\title{
Modelling Dynamic Decision Making with the ACT-R Cognitive Architecture
}

\author{
David Peebles \\ Department of Behavioural and Social Sciences, \\ University of Huddersfield, \\ Queensgate, \\ Huddersfield, \\ HD1 3DH, UK
}

D.PEEBLES@HUD.AC.UK

\section{Adrian P. Banks}

A.BANKS@SURREY.AC.UK

Department of Psychology,

Faculty of Arts and Human Sciences,

University of Surrey,

Guildford,

Surrey,

GU2 $7 X H, U K$

Editor: Pei Wang

\begin{abstract}
This paper describes a model of dynamic decision making in the Dynamic Stocks and Flows (DSF) task, developed using the ACT-R cognitive architecture. This task is a simple simulation of a water tank in which the water level must be kept constant whilst the inflow and outflow changes at varying rates. The basic functions of the model are based around three steps. Firstly, the model predicts the water level in the next cycle by adding the current water level to the predicted net inflow of water. Secondly, based on this projection, the net outflow of the water is adjusted to bring the water level back to the target. Thirdly, the predicted net inflow of water is adjusted to improve its accuracy in the future. If the prediction has overestimated net inflow then it is reduced, if it has underestimated net inflow it is increased. The model was entered into a model comparison competition - the Dynamic Stocks and Flows Challenge - to model human performance on four conditions of the DSF task and then subject the model to testing on five unseen transfer conditions. The model reproduced the main features of the development data reasonably well but did not reproduce human performance well under the transfer conditions. This suggests that the principles underlying human performance across the different conditions differ considerably despite their apparent similarity. Further lessons for the future development of our model and model comparison challenges are considered.
\end{abstract}

Keywords: ACT-R, Cognitive Architecture, DSF, Dynamic Decision Making.

\section{Dynamic decision making}

The term Dynamic decision making (DDM) covers a wide range of relatively complex cognitive tasks in which people must make a number of decisions over a period of time in a changing task environment. Environmental changes can depend on the structure of 
the environment itself, on decisions made by the decision maker or, most typically, some combination of the two (Brehmer, 1992; Edwards, 1962). Many real-world tasks require some form of DDM, ranging from game playing and car driving to patient care management, military battlefield strategy and economic market analysis.

Experimental studies of DDM have typically taken the form of simulated task environments in which participants are required to monitor certain states of the environment (often represented numerically or graphically) and maintain or optimise the value of one or more other states over a period of time (see Gonzalez, Vanyukanov, and Martin, 2005, for a review). Results from such studies have shown that people typically find such tasks challenging and often perform sub-optimally (e.g., Brehmer and Allard, 1991; Kleinmuntz and Thomas, 1987; Sterman, 1989).

There have been several proposed explanations for observed sub-optimal behaviour which fall roughly into three categories. The first argue that sub-optimal performance can result from constraints on processing imposed by the structure or properties of the cognitive system itself, such as working memory capacity. For example, in an early study of human DDM ability, Rapoport (1966) was able to account for sub-optimal performance with a model that assumed people had limited capacity to plan ahead. More recently, Gonzalez, Thomas, and Vanyukov (2005) found positive correlations between performance on three DDM tasks and score on the Visual-Span Test measure of working memory capacity (Shah and Miyake, 1996) and the Raven Progressive Matrices measure of fluid intelligence (Raven, 1962, 1977).

An alternative explanation for sub-optimal performance is that people construct incomplete or incorrect mental models of the task (e.g., Besnard, Greathead, and Baxter, 2004; Brehmer, 1992; Sterman, 1994). Problem solvers are assumed to construct a mental model prior to, and during the course of, interacting with a task environment, which consists of a representation of the relevant variables, their given properties and relationships, and hypotheses about relationships not given. Dynamic decision environments vary in terms of their complexity (e.g., the number of interacting elements and the functions underlying their interactions) and opacity (the degree to which elements and their relationships are hidden from the problem solver) which affects the accuracy and completeness of any mental models constructed. In addition, mental models about hidden relationships may have to be constructed using limited feedback information from one or two observed variables.

The third proposal is that sub-optimal performance results from sub-optimal strategies employed by problem solvers. A number of studies have shown, for example, that problem solving performance is related to the systematicity of the exploration strategy adopted, with more systematic strategies (i.e., exploring the properties of individual variables consecutively) resulting in richer structural knowledge and better performance (e.g., PutzOsterloh, 1993; Vollmeyer, Burns, and Holyoak, 1996).

Of course, these factors are not mutually exclusive and are bound to be related; problem solvers' strategies are more likely to be incorrect if they have inappropriate mental models of the task. The complex interaction of factors makes attempting to provide mechanistic, computational accounts of human DDM behaviour challenging if structural knowledge, strategic knowledge, learning and cognitive architectural constraints are all to be taken into account. 
One promising - and increasingly popular - way to formulate hypotheses about the cognitive representations and processes involved in DDM is to construct task models in a cognitive architecture. Cognitive architectures are computational theories of the large-scale structure of the mind; how cognition is controlled and how knowledge is encoded, stored, retrieved and utilised. There is a wide variety of cognitive architectures in existence, with different representational and processing assumptions, and (to a greater or lesser extent) informed by psychological theory. The advantage of many of these theories is that they are implemented as software systems in which task models can be specified and tested by running simulations and comparing model output with human data.

Currently all of the most prominent symbolic architectures (e.g., ACT-R (Anderson et al., 2004; Anderson, 2007), EPIC (Meyer and Kieras, 1997), and Soar (Laird, Newell, and Rosenbloom, 1997)) are built around some form of production system, a computational formalism that has been used to account for performance in DDM tasks for a number of years (e.g., Anzai, 1984). The architectures agree on other issues in addition to this common processing mechanism but also differ in terms of their assumptions concerning matters such as memory representation, conflict resolution, serial versus parallel processing etc.

The benefit of using cognitive architectures to model human performance in DDM tasks is that they allow one to specify the internal model of the dynamic system assumed to be held by the problem solver, the strategy being adopted and the precise nature of the computational processes being carried out on environmental information. All cognitive architectures incorporate one or more learning mechanisms while some (ACT-R and EPIC for example) are also highly constrained by psychological theories of learning, memory and visual processing and so provide more plausible accounts of human data. In the following section we provide a brief overview of ACT-R, the architecture employed in this study.

\subsection{The ACT-R cognitive architecture}

ACT-R is the current version of a proposed unified theory of cognition (Newell, 1990) developed by John Anderson and his colleagues over a period of 30 years (Anderson et al., 2004; Anderson, 2007). It consists of a set of independent modules that acquire information from the environment, process information and execute motor actions in the furtherance of particular goals. Figure 1 illustrates the main components of the architecture. There are four modules that comprise the central cognitive components of ACT-R. Two of these are memory stores for two types of knowledge: a declarative memory module that stores factual knowledge about the domain, and a procedural memory module that stores the system's knowledge about how tasks are performed. The former consists of a network of knowledge chunks while the latter is a set of production rules of the form "IF < condition $>$ THEN <action>": the condition specifying chunks that must be present for the rule to apply and the action specifying the actions to be taken should this occur.

Two further cognitive modules represent information related to the execution of tasks; a control state module keeps track of the intentions of the system and a problem state module maintains the current state of the task during problem solving.

In addition to the cognitive modules, four perceptual-motor modules for speech, audition, visual and motor processing encode perceptual information from the environment and enact speech or motor output. The visual and motor modules (shown in Figure 1) 


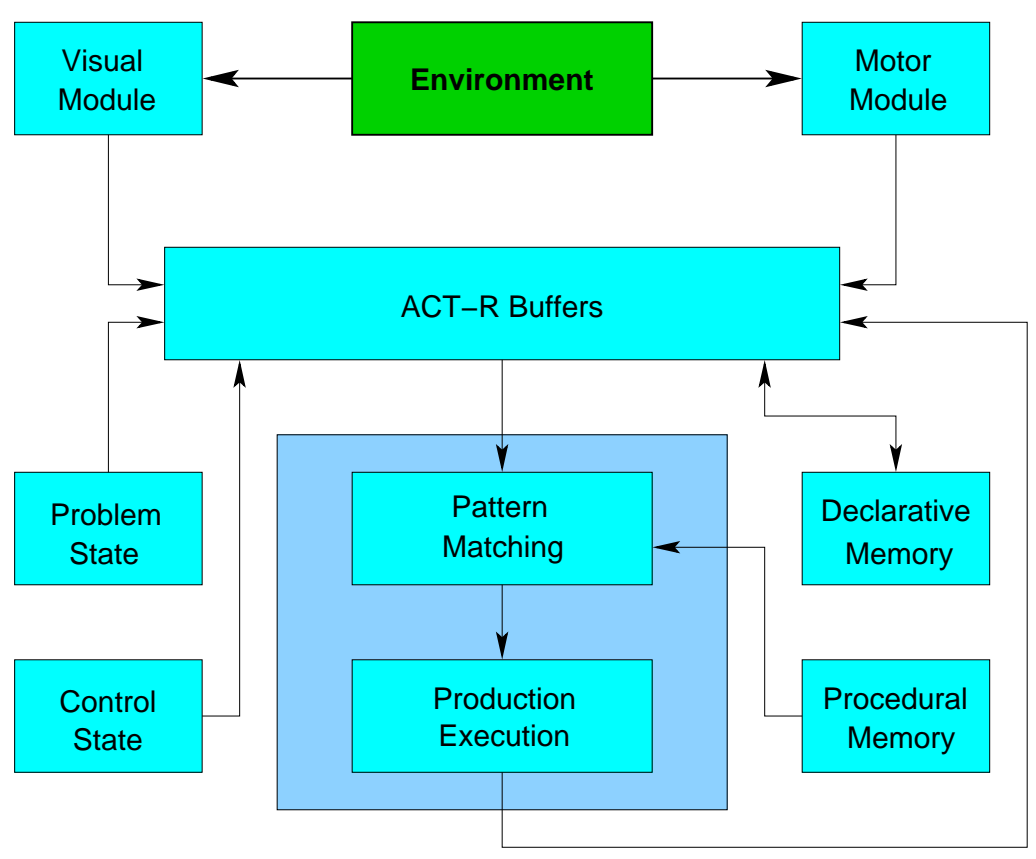

Figure 1: The modular structure of ACT-R 6.0

provide ACT-R with the ability to simulate visual attention shifts to objects on a computer display and manual interactions with a computer keyboard and mouse.

Each of ACT-R's modules has an associated buffer that can hold only one chunk of information from its module at a time and the contents of all of the buffers constitute the state of an ACT-R model at any one time. Cognition proceeds via a pattern matching process that attempts to find production rules with conditions that match the current contents of the buffers. When a match is found, the production "fires" and the actions (visual or manual movements, requests for the retrieval of a knowledge chunk from declarative memory, or modifications to buffers) are performed. Then the matching process continues on the updated contents of the buffers so that tasks are performed through a succession of production rule firings.

The processing in ACT-R's modules is serial but the modules run in parallel so that the system can move visual attention while also moving the mouse and attempting to retrieve knowledge from declarative memory. ACT-R processes also have associated latency parameters taken from the psychology literature. For example, it typically takes $50 \mathrm{~ms}$ for a production to fire and the time taken to move the mouse cursor to an object on the computer screen is calculated using Fitts' Law (Fitts, 1954).

In addition to these symbolic level mechanisms, ACT-R also incorporates a subsymbolic (i.e., numerical) level of computations that govern memory retrieval and production rule selection. The retrieval mechanism is based on the notion of activation; a chunk in declarative memory has a level of activation which determines its availability for retrieval, the level of which reflects the recency and frequency of its use. This allows models to account for widely observed recency and frequency effects on retrieval and forgetting. Subsymbolic computations also govern the probability of productions being selected in 
the conflict resolution process. At its most simple, it is assumed that people choose the most efficient actions (i.e., those that maximise the probability of achieving the goal in the shortest amount of time). In addition, the more often a production is involved in the successful achievement of a goal, the more likely it will be selected in the future.

ACT-R can be used to implement several different modelling paradigms (see Taatgen, Lebiere, and Anderson, 2006, for a review) and is the basis for the instance based learning theory (IBLT) model of DDM (Gonzalez, Lerch, and Lebiere, 2003; Martin, Gonzalez, and Lebiere, 2004). In the following sections we describe the dynamic stocks and flows DDM task and then a proposed ACT-R model of the task.

\section{The DSF task and challenge}

The DSF challenge was to create a cognitive model of a DDM task, and then compare the performance of this model against human performance in five experimental conditions that were not revealed to the competitors until the models had been submitted. The motivation behind this challenge was to stimulate a comparison between different cognitive models and architectures in order to identify their strengths and weaknesses, an approach that has been successfully applied previously (Gluck and Pew, 2005). To do this a task was required which would provide a rich testbed for complex cognition but also lend itself to a range of cognitive modelling approaches. A DDM task was chosen to fit these criteria.

The DDM task used in the challenge was the dynamic stocks and flows (DSF) task (Dutt and Gonzalez, 2007; Gonzalez and Dutt, 2007). This task is designed to investigate how people manage stocks and flows in a changing environment and takes the form of a "microworld" (Gonzalez, Vanyukanov, and Martin, 2005; Gray, 2002) comprised of simple components. These are: the level of stock $(S)$ within the system; an inflow which increases the stock; and an outflow which decreases the stock. The inflow and outflow are both comprised of an environmental component, which is determined by the system (EI and $E O$ respectively), and a user component, which is determined by the participant (UI and $U O$ respectively). Therefore participants have partial control over the inflow and outflow of stock. The stock simulated in the task is water. Water flows into the tank at a rate determined by $E I$ and $U I$, and flows out again at a rate determined by $E O$ and $U O$. The stock level at time $t$ is defined by

$$
S_{t}=S_{t-1}+\left[E I_{t-1}+U I_{t-1}\right]-\left[E O_{t-1}+U O_{t-1}\right]+S L_{t-1-d}
$$

where $S L$ is the supply line and $d$ is the delay in the supply line so that changes to $U I$ and $U O$ have an effect on the system only after a certain time period rather than immediately. The goal of the task is to maintain the amount of water in the tank at a specific level whilst the rate of water flow in and out changes over time.

There were two factors in this task which were controlled by the challengers to create a number of different conditions. These were (a) the changing rate of environmental flow into the tank, and (b) the delay between the $U I$ and $U O$ and their effect on the system. Both of these factors were manipulated in the challenge. Human data from two experiments in which participants carried out the four conditions of the DSF task (Dutt and Gonzalez, 2007; Gonzalez and Dutt, 2007) were made available when the challenge was initially announced as 
a basis for model development, (henceforth referred to here as the development conditions). Each of the four conditions ran for 100 time periods and had a different rate of EI:

1. Linear increasing $(0.08 \times($ TimePeriod $)+2)$,

2. Linear decreasing $(0.08 \times($ TimePeriod -1$)+10)$,

3. Nonlinear increasing $(5 \times \log ($ TimePeriod $))$

4. Nonlinear decreasing $(5 \times \log (101-$ TimePeriod $))$.

The mean human (and model) performance data are shown in Figure 2. Note that to facilitate comparison between the observed and model data for the different conditions, the upper range of each y axis has been adjusted to fit the maximum value.

The data reveal that people are generally able to maintain the tank at, or close to, the goal level after an initial learning period of varying length where the tank level is above the desired level. The data also show that for both linear and nonlinear cases, individuals learn to control the system more quickly for the positive functions than the negative ones (Dutt and Gonzalez, 2007).

\section{An ACT-R model of the DSF task}

From an analysis of the human performance data, we identified a number of key features which we assumed could be relevant for the development of a cognitive model. The first, previously noted by the challenge organisers (e.g., Dutt and Gonzalez, 2007), is that control over the system gradually improves over the first ten to thirty time periods to a state where accuracy levels are relatively stable and tank levels are generally close to target.

It is reasonable to assume that in the early time periods people are learning how the system operates and making initial hypotheses about the relationships between the variables. Once subjects have constructed a mental representation of the system, the monotonic and incremental nature of the functions means that subjects are generally able to maintain the tank at the target level (although close examination of individual performance profiles revealed substantial individual differences in the time taken to achieve and sustain target tank levels). The second feature of the data from the early time periods (particularly in the decreasing conditions) is that the tank is overfull and that subjects must reduce rather than increase the level to the target.

One cause of overfilling is if the amount of $E I$ is underestimated, which leads to an underestimate in the $U O$ necessary to stabilise the water level. One plausible explanation for this underestimate lies in the initial assumptions that participants make when starting the task. There are few cues in the DSF task environment about what the inflow is likely to be. At the first time period participants know only that the current water level is 0 , the target level is 4 and, crucially, that the scale on the display used to indicate water level runs from 0 to 10. This final observation provides parameters for the likely water level because it is reasonable (although in this case incorrect) to assume that the display will be designed to show likely water levels.

In the decreasing conditions, $E I$ in the first time period fills the tank completely. Unless $U O$ is set to remove almost all of this water, the tank will overflow in the second time 


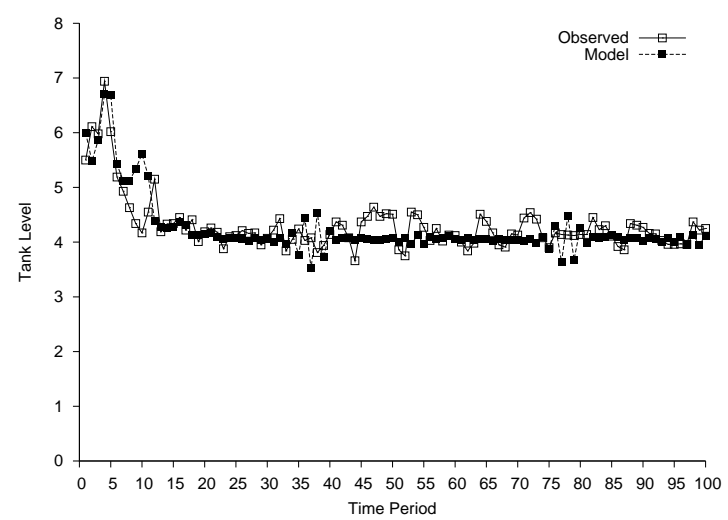

(a) Linear Increasing

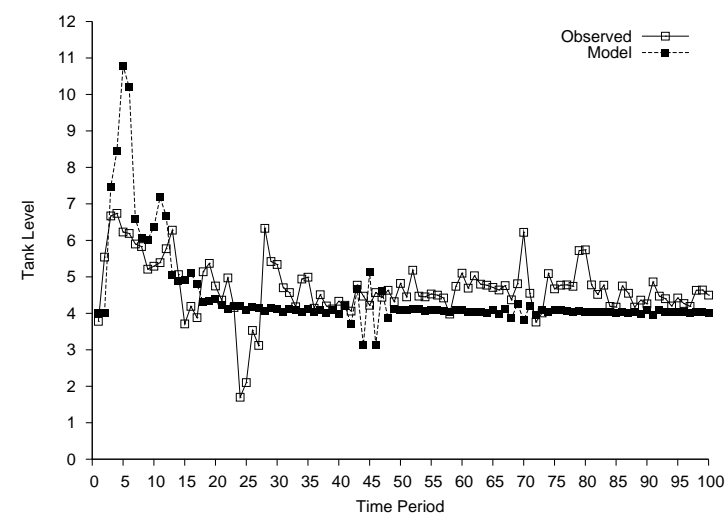

(c) Nonlinear Increasing

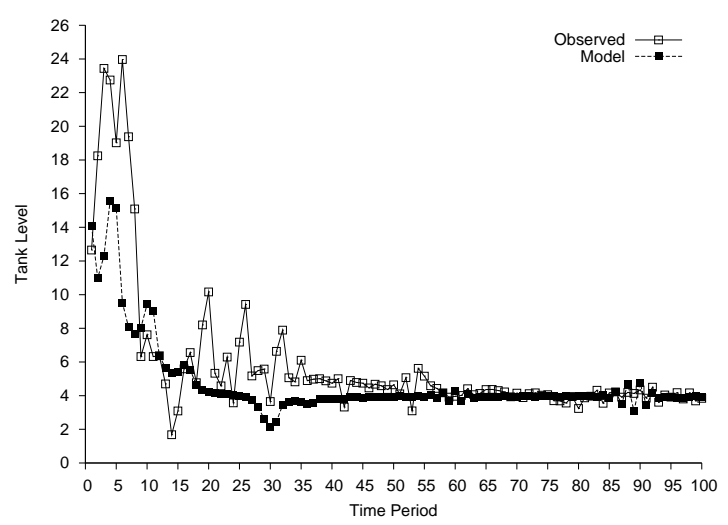

(b) Linear Decreasing

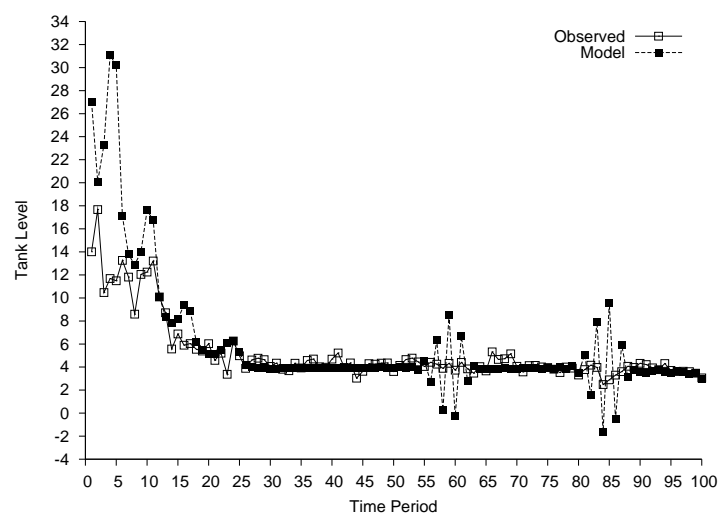

(d) Nonlinear Decreasing

Figure 2: Observed and ACT-R model performance in the DSF development conditions.

period. Thus, the expectations set by the experiment lead to an underestimate of the initial levels of $E I$ in the decreasing conditions. These assumptions are not faulty in the increasing conditions in which the initial $E I$ is around 2, a more reasonable figure to expect if you are assuming the approximate level will be between 0 and 10. On this basis, it is reasonable to expect that the decreasing conditions will initially overflow considerably as $E I$ is higher than expected whereas the increasing conditions will not as $E I$ is not unexpectedly high. Further, a greater overfilling would be expected in the nonlinear decreasing condition because EI remains higher for longer. The patterns of data shown in Figure 2 support these explanations.

We also suggest that the tank level range in the DSF display may also contribute to an explanation of why participants were generally slow to react to the high water levels by failing to increase the user outflow sufficiently (it typically takes subjects between ten to thirty time periods to reduce the level to the target value). As noted above, the DSF display runs from zero to ten and any level of water above ten is simply displayed as a full tank. When this occurs therefore, participants are unable to use this visual cue to obtain information about the water level in the tank. This may lead them simply to keep increasing 
the value of $U O$ until the level goes below ten or learn to attend to the less salient numeric tank level display. In addition, it is reasonable to assume that it may take more than the evidence of one or two time periods for participants to adjust their faulty assumptions.

The basis of the model then is that participants learn the EI based on the amount of water flowing into the tank. They initially underestimate what this level will be however, and are slow to adjust their estimates in the direction of the correct value. This explanation accounts for the main features of the group level data in the development conditions.

\section{Assumptions of the model}

The DSF task environment presents six variables to the problem solver: Environmental Inflow (EI), Environmental Outflow (EO), User Inflow (UI), User Outflow (UO), Current tank Level (CL) and Goal Level (GL). These are presented to cognitive models directly as numerical values over a socket connection.

Our ACT-R model of the DSF task consists of a set of eleven ACT-R production rules. Figure 3 displays the productions (shown as rectangles) and the flow of control between them. The model assumes that four items of declarative knowledge are maintained and employed in carrying out the task: (a) the estimated net environmental input to the tank, (b) the current difference $\left(d_{c}\right)$ between the actual and target tank levels, (c) the previous difference $\left(d_{p}\right)$ between the actual and target tank levels in the previous trial, and (d) the rate of change $(m)$ of the adjustment for the estimated net environmental change.

The strategy embodied by the model can be divided into three main steps. In the first step the model estimates the net environmental input for the next time period, based on the accuracy of the prediction for the previous one. For each time period, the estimate for the net environmental input in the following time period is the current net environmental input plus the model's estimate of the change in the net environmental input. This estimated change is the difference between the current tank level and the target level (i.e., the error in the previous prediction) multiplied by the model's estimate of the rate at which the net environmental input is changing. These estimates are carried out by the test difference production.

The model assumes that problem solvers must attempt to keep a track of how the net environmental input is progressing over time periods so that the rate at which the net user change $(U I-U O)$ changes can be kept the same as $m$. A major component of the model, therefore is the mechanism to adjust the estimated rate at which the net environmental input is changing at each time period, $m_{t}$. If $d_{c}$ is greater than $d_{p}$, then the rate of the net user change is insufficient (i.e., $E I$ and $E O$ are changing faster than the estimate). Therefore the model's estimate of $m$ must be increased so that the net user change is adjusted in line with the actual changes to $E I$ and $E O$. Specifically, the rate of change is increased according to how much more $d_{c}$ is greater than $d_{p}$. A large relative increase leads to a large increase in rate of change and so on, calculated according to the equation

$$
m_{t+1}=m_{t}+\theta \frac{\left|d_{c}\right|+\left|d_{p}\right|}{\left|d_{c}\right|} .
$$




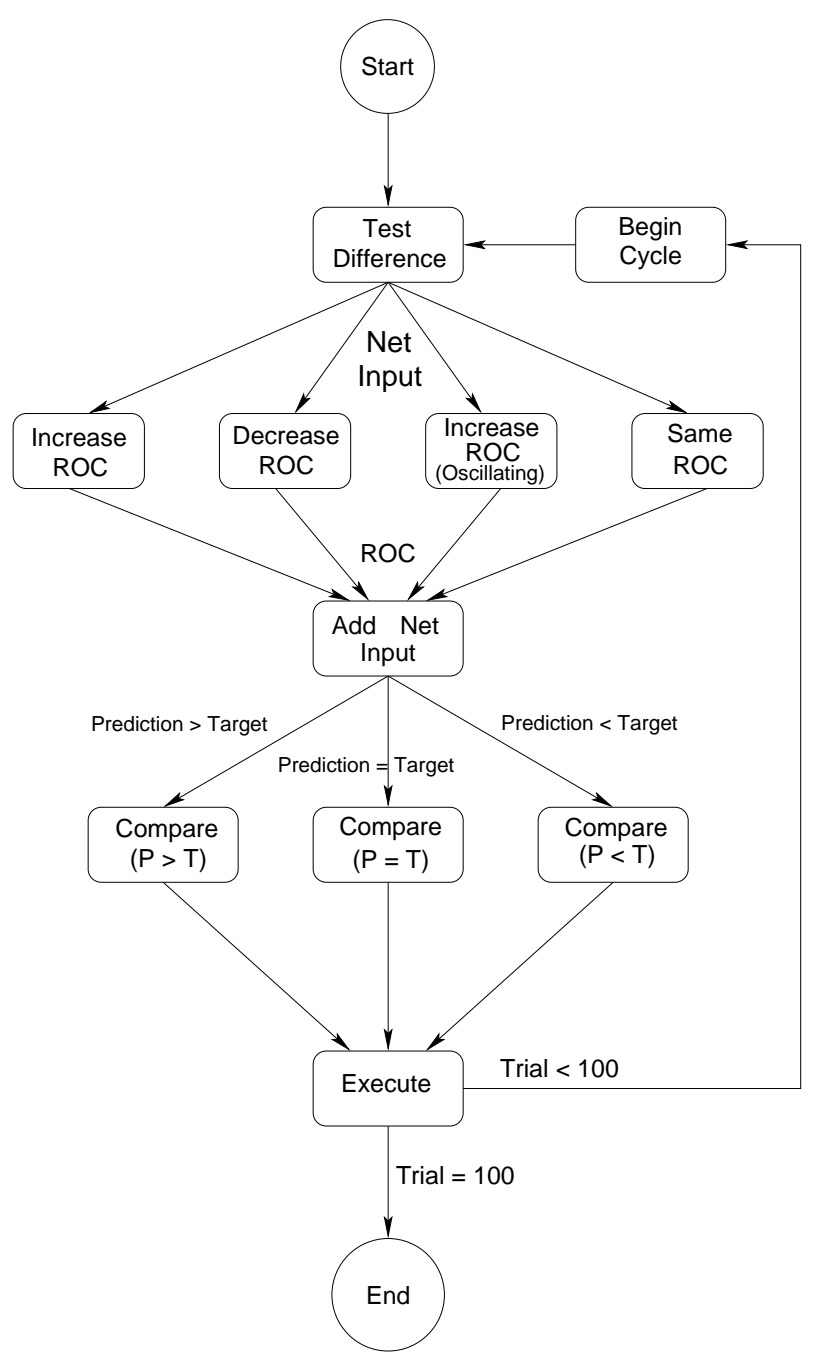

Figure 3: Control flow of the model.

where $\theta$ is a parameter representing an estimate of people's accuracy at adjusting the rate of change. If $\theta=1$ then the model's adjustment is perfect whereas a value of 0.5 was found to capture the human data well by under-adjusting the rate of change.

In the second step (implemented by the four $R O C$ productions), the model calculates the rate of change according to the relationship between the current difference and that from the previous time period. If the current difference is greater than the previous difference the rate of change is increased accordingly. Alternatively, if the difference is decreasing (either positively or negatively), the model decreases the rate of change in proportion whereas if the difference is constant then the model keeps the current rate of change constant. An additional production was also added to manage situations where the rate of change fluctuates either side of the goal. In this case the difference between the current and target levels is not a reliable indicator of the accuracy of the previous estimate and the rate of change is reduced to prevent an ever-increasing rate of change. 
In the third step the model predicts the next tank level by adding the net environmental input to the current tank level (the add net input production). Once this has been estimated, the model then simply adjusts $U I$ or $U O$ by the difference between the predicted next level and the target level to bring the level to the target. This is handled by three compare productions, which fire depending on the relative values of the predicted and target levels. Finally the execute sends the decision to the DSF simulation.

The three-step process can be further illustrated by an example. For a particular time period, $t$, if the current tank level is 6 , the target level is 4 , and the current estimate of the rate of change, $m_{t}=1$, then $d_{c}=2$ and the model computes the net environmental input as $m_{t} \times d_{c}=2$. In addition, assuming $d_{p}=1$ then the error in the model's prediction is increasing so the model adjusts its estimate of the rate of change $m_{t+1}$ to be $1+0.5 \frac{|2|+|1|}{|2|}=$ 1.75 (as the size of the difference has increased across time periods so has the model's estimate of the rate of change).

The predicted level for $t+1$ therefore is the current level plus the net environmental input $(6+2=8)$. The model will then compensate for this predicted discrepancy by setting $U I=0$ and $U O=8-4=4)$.

As can be seen in Figure 2, the performance of the model in the four development conditions is reasonably close to that of the human experiment participants. The goodnessof-fit measures used for the challenge $\left(R^{2}\right.$ and $\left.R M S E\right)$ for the model are shown in Table 1.

Table 1: $R^{2}$ and RMSE goodness-of-fit measures for the ACT-R model on the four development conditions

\begin{tabular}{ccccc}
\hline & \multicolumn{4}{c}{ Development Condition } \\
\cline { 2 - 5 } Error & Linear Inc. & Linear Dec. & Nonlinear Inc. & Nonlinear Dec. \\
\hline$R^{2}$ & 0.63 & 0.67 & 0.26 & 0.74 \\
$R M S E$ & 0.34 & 2.97 & 1.06 & 3.7 \\
\hline
\end{tabular}

\section{Testing the model}

The DSF challenge was designed not simply to test models' ability to account for the given data sets but primarily to test their ability to predict new, unseen data sets from five different conditions of the DSF task after they bad been constructed. No information about the nature of these new transfer conditions was made available during the model construction phase. The stated aim of this approach was that, because model performance in the test phase could not be modified by hand, to predict the transfer data accurately, models must capture general principles governing human performance in the DSF task rather than specific features of a single condition or data set.

The five conditions against which the submitted models were tested were not simple variations of these, such as different linear or nonlinear functions; they were qualitatively different. Three conditions manipulated EI according to a repeated sequence for the 100 trials:

1. $S_{2} . E I$ is the sequence 1,5 . 
2. $S_{2} \pm 1$. $E I$ is the sequence $1 \pm 1,5 \pm 1$, with \pm 1 noise being distributed equally amongst trials.

3. $S_{4}$. EI is the sequence $0,4,2,6$.

The two other transfer conditions manipulated the delay between the submission of users' $U I$ and $U O$ decision and its execution:

1. $D_{2} . U I$ and $U O$ decisions were delayed until the trial after submission.

2. $D_{3} . U I$ and $U O$ decisions were delayed until two trials after submission.

Both delay conditions started with 4 gallons of water, a goal level of 6 , and $E O$ set at 0 . The $E I$ function was a linear increasing function that deposited water into the tank from 2 to 10 gallons over the course of 100 trials.

The human and model data for the five transfer conditions are displayed in Figure 4. Note that, as in Figure 2, the upper range of each y axis has been adjusted to aid comparison. In this case, the upper bound has been set to allow a reasonable display of the pattern of values, even if this required omitting extreme cases. The $R^{2}$ and $R M S E$ goodness-of-fit measures for the model are shown in Table 2.

There are a number of comments to make about the data in Figure 4. Firstly, it can be seen that the human data does not resemble that from the development conditions but varies widely between transfer conditions. Overall, subjects performed less well in all five transfer conditions than in the development ones, most likely because the underlying functions were less easy to discern. This could be because, unlike the relatively simple monotonic functions in the development conditions in which only the rate and direction of $E I$ was manipulated, the transfer functions were either non-monotonic, contained noise, affected the control of the system, or required the taking of several time periods into account.

Although there is some degree of similarity between the patterns in the three sequence conditions, they differ considerably from the two delay conditions. All three sequence conditions display a regular "saw tooth" pattern reflecting the repeated sequence pattern and a general overfilling of the tank. The $S_{2}$ condition does show a pattern of overfilling and then stabilising like the development conditions (although with less accuracy as it overfills every other time) whereas $S_{2} \pm 1$ displays a series of over-corrections in which the tank is overfilled but never stabilises. The $S_{4}$ condition also appears to be overfilled but moving towards stabilising towards the goal level to some extent, although very slowly so that the target is not achieved before the end of the experiment. In addition, it seems that the tank overfills every time there is a large input.

Human performance was somewhat similar for the $D_{2}$ and $D_{3}$ conditions; both show an overfilled tank with a series of over-corrections, the pattern being more varied and less accurate in the latter.

The model did not capture human performance in any of the five transfer conditions very well. For all three sequence conditions the tank level tended to oscillate around the correct with periods of large over-correction. Model performance in the delay conditions was also quite dissimilar to human data. In the $D_{2}$ condition, model performance matches human performance very closely for the first 15 time periods but then the tank level increases 


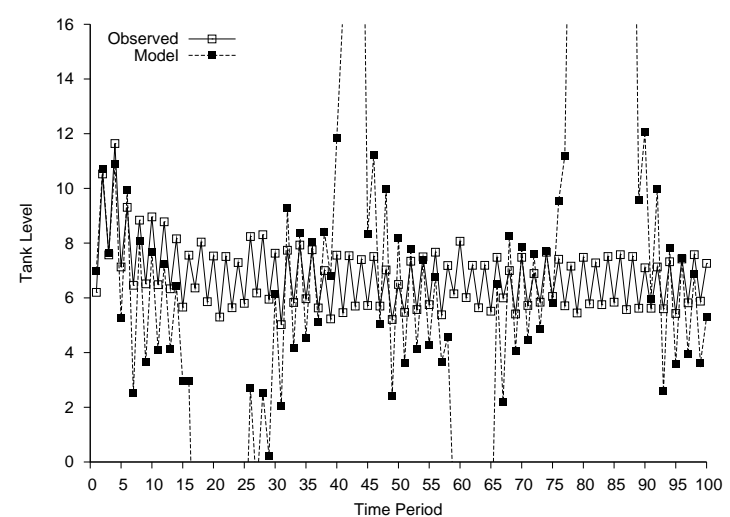

(a) Sequence 2

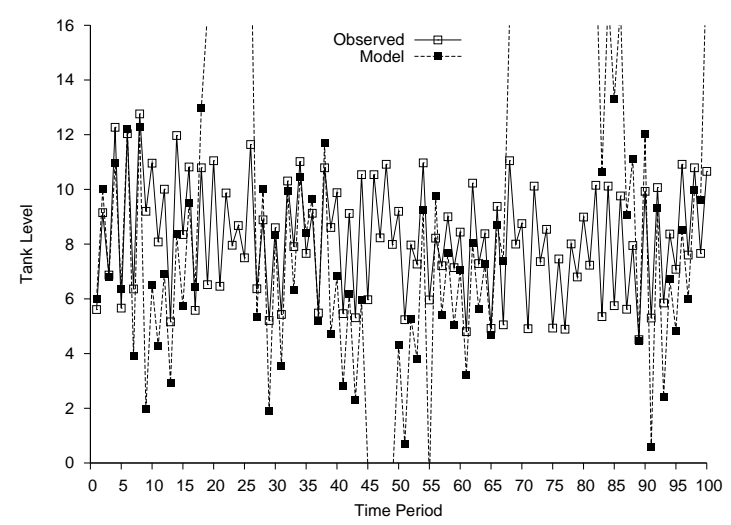

(b) Sequence 2 plus noise

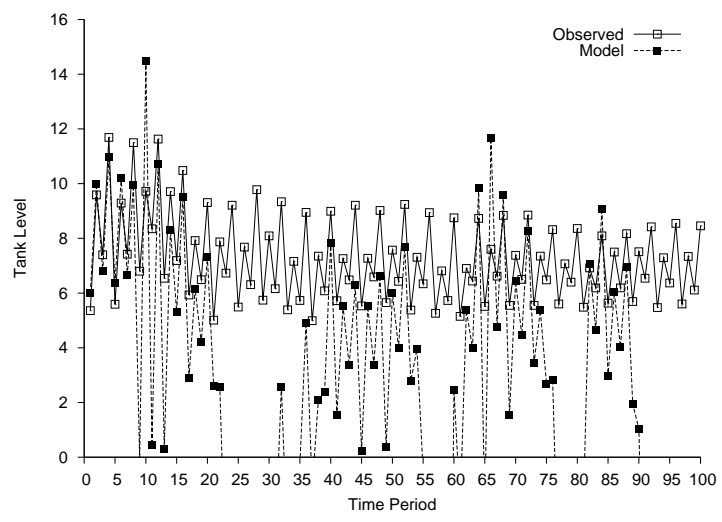

(c) Sequence 4

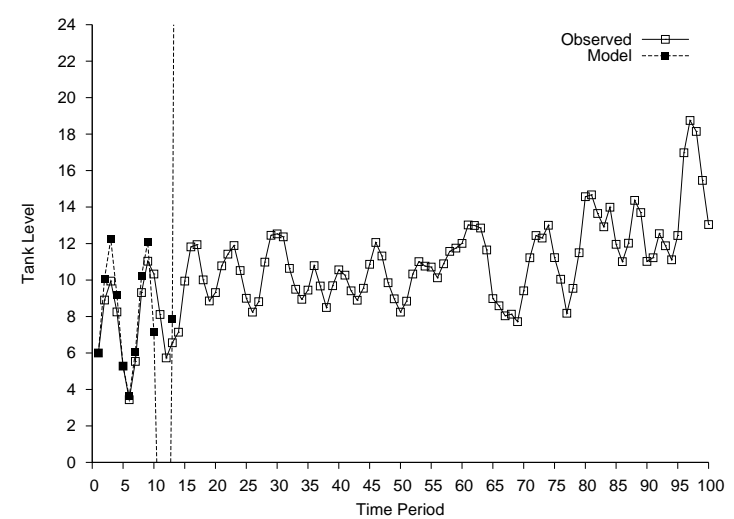

(d) Delay 2

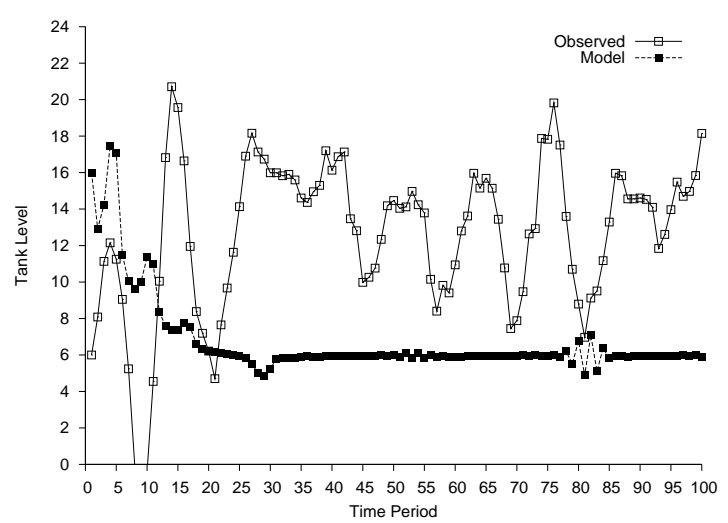

(e) Delay 3

Figure 4: Observed and ACT-R model performance in the DSF transfer conditions.

rapidly and never returns to normal levels. In the $D_{3}$ condition, the model is able to perform in a similar fashion to the development conditions, showing the familiar pattern of overfilling and then stabilising at the correct value. However, this is not what people do. 
Table 2: $R^{2}$ and $R M S E$ goodness-of-fit measures for the ACT-R model on the five test conditions

\begin{tabular}{cccccc}
\hline & \multicolumn{5}{c}{ Test Condition } \\
\cline { 2 - 6 } Error & $S_{2}$ & $S_{2} \pm 1$ & $S_{4}$ & $D_{2}$ & $D_{3}$ \\
\hline$R^{2}$ & 0.16 & 0.4 & 0.5 & 0.02 & 0.11 \\
$R M S E$ & 0.96 & 0.38 & 0.59 & 0.53 & 1.25 \\
\hline
\end{tabular}

We are unable to provide a general explanation of the model's behaviour in the transfer conditions or more specifically for the delay conditions other than to assume that it has something to do with the fact that the model only takes the previous trial into account when adjusting the estimated rate at which the net environmental input is changing at each time period. For several of these conditions, it may be the case that accurate prediction relies on the encoding and integration of information from a number of previous trials.

\section{Conclusions}

The process of developing a cognitive model of a complex task is challenging if one seeks to generate accurate fits to human data over a wide range of different task scenarios. It requires deep insights into the three factors that have been identified as affecting performance: the strategy or strategies employed, the various constraints imposed by the cognitive system, and the task representations constructed and used by the problem solver. The ACT-R cognitive architecture is well suited for modelling such tasks because it combines a symbolic level for modelling knowledge structures and strategic, decision making processes with a subsymbolic level for modelling constraints such as working memory capacity, learning and forgetting rates etc.

It is not necessary to use all of the available mechanisms in ACT-R however and in developing this model we have chosen to focus exclusively on the strategic processes. In terms of the space of possible models therefore, the model is relatively minimal and abstract; it is essentially an implementation of a relatively high-level specification of the strategy we hypothesised subjects employed. We did not utilise ACT-R's subsymbolic learning or memory retrieval mechanisms, nor did we model the details of the various calculations that were required. To the extent that the model captured the given data however, we can say that the assumptions of the model were supported. We believe that the algorithm suggested may well lie at the core of a more sophisticated and elaborate model that captures the human data more closely - from the developmental conditions at least.

There are several possible options for making the model more complex. For example, during each trial of the task, the current model creates a single declarative knowledge structure containing the four required items of knowledge and maintains them in ACT-R's imaginal buffer throughout the trial. The model therefore is not required to retrieve any of this knowledge during the trial or seek it from the environment as part of a strategy or if a retrieval failure occurs. Although this is convenient and sufficient for our current purposes, it no doubt glosses over possibly important processes and a more detailed model would probably represent these knowledge elements separately (in the current model, if the 
chunk is forgotten then all four knowledge items are irretrievable), and would be required to recall the knowledge (or seek it from the environment) when required.

The second type of explanation for suboptimal human performance revolves around the accuracy of the mental models constructed during the task. For the DSF task, such a mental model will consist of a representation of the variables and their values, given relationships between variables, and hypotheses about relationships not given. The DSF task environment inputs the six variables (EI, EO, UI, UO, CL, and GL) to the ACT-R model directly as numerical values and the mental model of their relationships is embodied primarily in the set of numerical calculations employed by the model to compute the values of the declarative knowledge elements.

Although we believe that the mental model we have hypothesised is a reasonably accurate characterisation of that used by most human participants, for the sake of simplicity and in order to allow us to focus on strategic considerations, the various numerical calculations and comparisons carried out by the current model were not implemented using cognitive mechanisms (i.e., memory retrievals or production based computations), but were simply implemented as Lisp code in productions. A more fine-grained model would replace these substitute functions with more plausible psychological mechanisms.

The third proposed explanation for suboptimal human performance relates to the strategies employed to complete DDM tasks. In ACT-R models, problem solving strategies are represented by the control structure embodied in the model's production rule set. A single model may contain productions to allow several strategies for the same task with the goal of the modelling enterprise being to use ACT-R's production rule utility learning mechanism to account for the learning and selection of different strategies over the course of problem solving (e.g., Lovett and Anderson, 1996, 2005).

The generally close fit of the model to the human data in the developmental conditions suggests that the strategy we have proposed is a reasonable first start and a good basis for further elaboration. It is probably not the only strategy available however and it may well be the case that alternative strategies were used by some of the participants - or that participants switched or modified strategies during the course of the experiment. One possible option for extending the current model therefore is to explore alternative strategies, implement them as ACT-R productions, test them as independent hypotheses, or combine them in a single model and let them compete for adoption using ACT-R's production rule utility learning mechanisms.

By using more of ACT-R's architectural mechanisms, the three options for extending the current model outlined above add further psychological constraints on the proposed explanation and, because they reduce the explicit control of the modeller, provide a more rigorous test of the architecture (one of the primary aims of the DSF challenge). In most cases the number of productions - and time - required to complete the task would increase, as would the likelihood of retrieval failures and errors (together with additional recalculations and search strategies required to obtain the information from the environment).

Such considerations of model accuracy and veracity could be taken even further by requiring the model to interact with the task environment at the eye movement, mouse movement and key-press level — an approach increasingly adopted by ACT-R modellers 
investigating interactive behaviour (e.g., Fleetwood and Byrne, 2006; Peebles and Cheng, 2003; Salvucci, 2006).

The question remains however, to what extent the current model would need to be modified in order to explain the transfer data. Although we have yet to test this, we believe that the performance of the model in a number of the transfer conditions is close enough to the human data to suggest that significant improvements to the fit could be achieved by relatively minor adjustments to parts of the model (e.g., the number of previous time periods taken into account) rather than a major revision of the proposed strategy. This assumes that the same factors are applicable to all of the scenarios being modelled, or at least that they are sufficiently similar that they can be captured in a single model that is not so general as to provide little psychological insight.

It may be the case, however, that more radical extensions to the current model in the ways outlined above are necessary in order to able to account for the transfer data adequately, for example by having a model that learns to adopt the best solution from a number of competing strategies and mental models. This approach would no doubt provide useful insights but suffers from the criticism that alternative strategies are simply being added in a post-hoc fashion by the modeller. Ultimately the optimal strategy for a model may be one in which one or more hypotheses are formed, tested, updated or abandoned during the course of the task.

The DSF challenge is an important and valuable exercise which should be lauded for its aims. In setting competitive opportunities for model comparison, modellers from different traditions and backgrounds are able to test their assumptions and methods against a set of external, objective criteria. The format of this challenge (model a given data set, test against novel, unseen data sets) also forces modellers to address the tension in modelling between the aims of specificity and closeness of fit to observed data and the generality of the model's assumptions.

To maximise the benefit of such endeavours in the future, however, it may be the case that a broader approach to model evaluation has to be taken, rather than simple closeness of fit as measured by $R^{2}$ and $R M S E$. Although these measures are useful as objective, quantitative criteria for assessing the relationship between model predictions and behavioural observations (cf. Roberts and Pashler, 2000), a more profitable (although admittedly more costly and time-consuming) enterprise would be to develop more sophisticated criteria for model evaluation in terms of the theoretical insight they provide about the task and the architecture used.

If $R^{2}$ and $R M S E$ are used as the sole criteria for model comparison, there is a danger that models will be produced that simply optimise these measures (while neglecting other factors such as parsimony) rather than models that provide genuine psychological insight. As this is antithetical to the long term aims of such challenges, we would argue that more appropriate criteria for conceptual understanding of human performance are necessary.

For example, in the DSF challenge, the organisers could have identified a number of qualitative features of human performance such as 'an initial overfilling of the tank before stabilising at the target' in the linear conditions; or 'a repeating larger overfill, return to target, smaller overfill, return to target pattern' in the $S_{4}$ condition and then assessed whether models showed these general features (irrespective of the exact quantity of water in the tank) as the criteria of success instead of (or more likely as well as) the other quantitative 
measures. Subsequently, as models improve and they all start to meet the initial goal of demonstrating the general features of human data, $R^{2}$ and $R M S E$ would presumably become more important as criteria.

These additional criteria may make it harder to identify a clear 'winner' but if the goal of such challenges is to work towards greater conceptual understanding then it may be found that several models are equal in terms of the insight they provide. This could, of course, take a longer time to assess than is typically set for such challenges and may only be known after further testing, comparison or integration. However the benefits of such an evaluation may far outweigh these costs.

This challenge has been a valuable exercise, progressing the tradition of earlier modelling challenges, and we hope that it will encourage and provide a useful example for future challenges. We also hope that the models entered into the challenge provide further insights into the DSF task and be of long-term benefit to the organisers. If lessons can also be learned from the challenge in terms of how models can be evaluated then the enterprise will have been doubly valuable, with consequences that have a long-term benefit for the cognitive modelling community.

\section{References}

Anderson, J. R.; Bothell, D.; Byrne, M. D.; Douglass, S.; Lebiere, C.; and Qin, Y. 2004. An integrated theory of the mind. Psychological Review 111:1036-1060.

Anderson, J. A. 2007. How can the Human Mind Occur in the Physical Universe? New York, NY: Oxford University Press.

Anzai, Y. 1984. Cognitive control of real-time event driven systems. Cognitive Science $8: 221-254$.

Besnard, D.; Greathead, D.; and Baxter, G. 2004. When mental model's go wrong: cooccurrences in dynamic, critical systems. International Journal of Human-Computer Studies 60:117-128.

Brehmer, B., and Allard, R. 1991. Real-time decision making: Effects of task complexity and feedback delays. In Rasmussen, J.; Brehmer, B.; and Leplat, J., eds., Distributed decision making: Cognitive models for cooperative work. Chichester: Wiley.

Brehmer, B. 1992. Dynamic decision making: Human control of complex systems. Acta Psychologica 81:211-241.

Dutt, V., and Gonzalez, C. 2007. Slope of inflow impacts dynamic decision making. In Eberlein, R. L.; Sterman, J. D.; and Oliva, R., eds., Proceedings of the 25th International Conference of the System Dynamics Society.

Edwards, W. 1962. Dynamic decision theory and probabilistic information processing. Human Factors 4:59-73.

Fitts, P. M. 1954. The information capacity of the human motor system in controlling the amplitude of movement. Journal of Experimental Psychology 47:381-391. 
Fleetwood, M. D., and Byrne, M. D. 2006. Modeling the visual search of displays: A revised ACT-R/PM model of icon search based on eye tracking data. Human Computer Interaction 21:153-197.

Gluck, K. A., and Pew, R. W., eds. 2005. Modeling Human Behaviour with Integrated Cognitive Architectures: Comparison, Evaluation and Validation. Mahwah, NJ: Lawrence Erlbaum Associates.

Gonzalez, C., and Dutt, V. 2007. Learning to control a dynamic task: A system dynamics cognitive model of the slope effect. In Lewis, R. L.; Polk, T. A.; and Laird, J. E., eds., Proceedings of the 8th International Conference on Cognitive Modeling, 61-66.

Gonzalez, C.; Lerch, F. J.; and Lebiere, C. 2003. Instance-based learning in real-time dynamic decision making. Cognitive Science 27:591-635.

Gonzalez, C.; Thomas, R. P.; and Vanyukov, P. 2005. The relationships between cognitive ability and dynamic decision making. Intelligence 33:169-186.

Gonzalez, C.; Vanyukanov, P.; and Martin, M. K. 2005. The use of microworlds to study dynamic decision making. Computers in Human Behaviour 21:273-286.

Gray, W. D. 2002. Simulated task environments: The role of high-fidelity simulations, scaled worlds, synthetic environments, and microworlds in basic and applied cognitive research. Cognitive Science Quarterly 2:205-227.

Kleinmuntz, D., and Thomas, J. 1987. The value of action and inference in dynamic decision making. Organizational Behavior and Human Decision Processes 39:341-364.

Laird, J. E.; Newell, A.; and Rosenbloom, P. S. 1997. SOAR: An architecture for general intelligence. Artificial Intelligence 33:1-64.

Lovett, M. C., and Anderson, J. R. 1996. History of success and current context in problem solving: Combined influences on operator selection. Cognitive Psychology 31:168-217.

Lovett, M. C., and Anderson, J. R. 2005. A strategy-based interpretation of Stroop. Cognitive Science 29:493-524.

Martin, M. K.; Gonzalez, C.; and Lebiere, C. 2004. Learning to make decisions in dynamic environments: ACT-R plays the beer game. In Lovett, M.; Schunn, C.; Lebiere, C.; and Munro, P., eds., Proceedings of the Sixth International Conference on Cognitive Modeling, 178-183.

Meyer, D. E., and Kieras, D. E. 1997. A computational theory of executive cognitive processes and multiple-task performance 1. Basic mechanisms. Psychological Review 104:3-65.

Newell, A. 1990. Unified Theories of Cognition. Cambridge, MA: Harvard University Press.

Peebles, D., and Cheng, P. C.-H. 2003. Modeling the effect of task and graphical representation on response latency in a graph reading task. Human Factors 45:28-46. 
Putz-Osterloh, W. 1993. Strategies for knowledge acquisition and transfer on knowledge in dynamic tasks. In Grube, G., and Wender, K. F., eds., The Cognitive Psychology of Knowledge. Amsterdam: Elsevier. 331-350.

Rapoport, A. 1966. A study of human control in a dynamic multistage dcision task. Behavioral Science 11:18-32.

Raven, J. 1962. Raven Standard Progressive Matrices Test. Oxford: Oxford Psychologists Press.

Raven, J. 1977. Advanced Raven Progressive Matrices. Oxford: Oxford Psychologists Press.

Roberts, S., and Pashler, H. 2000. How persuasive is a good fit? A comment on theory testing. Psychological Review 107:358-367.

Salvucci, D. D. 2006. Modeling driver behavior in a cognitive architecture. Human Factors $48: 362-380$.

Shah, P., and Miyake, A. 1996. The separability of working memory resources for spatial thinking and language processing: An individual differences approach. Journal of Experimental Psychology 125:4-27.

Sterman, J. D. 1989. Misperceptions of feedback in a dynamic decision making. Organizational Behavior and Human Decision Processes 43:301-335.

Sterman, J. D. 1994. Learning in and about complex systems. System Dynamics Review 10:291-330.

Taatgen, N. A.; Lebiere, C.; and Anderson, J. R. 2006. Modeling paradigms in ACT-R. In Sun, R., ed., Cognition and Multi-Agent Interaction: From Cognitive Modeling to Social Simulation. Cambridge: Cambridge University Press. 29-52.

Vollmeyer, R.; Burns, B. D.; and Holyoak, K. J. 1996. The impact of goal specificity on strategy use and the acquisition of problem structure. Cognitive Science 20:75-100. 